\title{
Oscillation of Nonlinear Fractional Dynamic Equations with Forcing Term
}

\author{
S. Manikandan $\mathbb{D D}^{1}$, V. Muthulakshmi $\mathbb{D}^{1},{ }^{1}$ S. Harikrishnan ${ }^{(D)}{ }^{2}$ \\ and Porpattama Hammachukiattikul ${ }^{3}{ }^{3}$ \\ ${ }^{1}$ Department of Mathematics, Periyar University, Salem 636 011, Tamilnadu, India \\ ${ }^{2}$ Department of Mathematics, Sona College of Technology, Salem 636 005, Tamilnadu, India \\ ${ }^{3}$ Department of Mathematics, Faculty of Science and Technology, Phuket Rajabhat University, Phuket 83000, Thailand
}

Correspondence should be addressed to Porpattama Hammachukiattikul; porpattama@pkru.ac.th

Received 10 November 2020; Accepted 9 August 2021; Published 18 August 2021

Academic Editor: Xian-Ming Gu

Copyright (c) 2021 S. Manikandan et al. This is an open access article distributed under the Creative Commons Attribution License, which permits unrestricted use, distribution, and reproduction in any medium, provided the original work is properly cited.

In this paper, interval oscillation criteria for the nonlinear damped dynamic equations with forcing terms on time scales within conformable fractional derivatives are established. Our approach is determined from the implementation of generalized Riccati transformation, some properties of conformable time-scale fractional calculus, and certain mathematical inequalities. Also, we extend the study of oscillation to conformable fractional Euler-type dynamic equation. Examples are presented to emphasize the validity of the main theoremslenleadertwodots.

\section{Introduction}

The consideration of dynamic equations on time scales has attracted many researchers because of its wide applications in the field of science and engineering. The theory of time scales was presented by Hilger [1] to unify the discrete and continuous analysis. It not only unifies the continuous and discrete cases but also gives new areas in between such as $q$-calculus [2]. The qualitative analysis of solutions of dynamic equations on different time scales has received considerable notice. In particular, the investigation of the oscillation of solutions to dynamic equations [3-5], dynamic equations with damping term [6-8], and dynamic equations on various time scales [9-11] has gained extensive attention. Fractional calculus is a generalization of integration and differentiation to any order. Recently, it has been realized that the fractional calculus has numerous applications in engineering, signal processing, economics and finance, probability and statistics, neural networks, and thermodynamics; see for illustrations [12-16] and the citations therein. In recent times, the importance has been given to fractional order calculus rather than integer order due to its applications in engineering such as neural networks, electrical and mechanical engineering, and population dynamics. The fractional dynamic equations on time scales have been studied by only few authors [17-19].

In [19], Feng and Meng established the asymptotic and oscillatory behavior of the following dynamic equation of fractional order on time scales using the generalized Riccati transformation technique:

$$
\begin{aligned}
& \left(a(t)\left(\left[r(t) x^{(\alpha)}(t)\right]^{(\alpha)}\right)^{\gamma}\right)^{(\alpha)}+p(t)\left(\left[r(t) x^{(\alpha)}(t)\right]^{(\alpha)}\right)^{\gamma} \\
& \quad+q(t) f(x(t))=0, \quad t \in\left[t_{0}, \infty\right)_{\mathbb{J}} .
\end{aligned}
$$

Alzabut et al. [17] considered the following nonlinear damped dynamic equation with a conformable fractional derivative:

$$
\begin{aligned}
& \left(r(t)\left(x^{(\alpha)}(t)\right)^{\gamma}\right)^{(\alpha)}+p(t)\left(x^{(\alpha)}(t)\right)^{\gamma}+q(t) f(x(t))=0, \\
& t \in\left[t_{0}, \infty\right)_{\mathbb{T}} .
\end{aligned}
$$


Here, the authors established the oscillation of the above equation when the nonlinear function $f$ is increasing and nonincreasing. Besides, the results are carried out in light of the following two cases: $\int_{t_{0}}^{\infty} \Delta^{\alpha} s / r(s)=\infty$ and $\int_{t_{0}}^{\infty} \Delta^{\alpha} s /$ $r(s)<\infty$. Motivated by the above discussion, in this work, we established the oscillation results of nonlinear conformable fractional dynamic equations with a forced term.

In the recent papers $[18,20]$, the conformable time-scale fractional calculus has been introduced. Applications of the obtained results demonstrate that the newly defined calculus will be applied to investigate oscillation for both fractional differential and fractional difference equations at the same time. Therefore, the determination of oscillation of solutions of conformable fractional dynamic equations has become a promising topic for researchers. To the best of our observation, papers $[17,19]$ are the only research that has studied the oscillation of conformable fractional dynamic equations.

\section{Problem Formulation and Preliminaries}

In this article, we are concerned with a class of nonlinear conformable fractional dynamic equations with damped and forced terms on time scales of the kind:

$$
\begin{aligned}
& \quad\left(\varphi(\eta)\left(y^{(\bar{\alpha})}(\eta)\right)\right)^{(\bar{\alpha})}+a(\eta) y^{(\bar{\alpha})}(\eta)+b(\eta) \mathbf{f}(y(\eta))=h(\eta), \\
& \eta \in\left[\eta_{0}, \infty\right)_{\widehat{\mathbb{\pi}}}
\end{aligned}
$$

where $\hat{\mathbb{T}}$ denotes an arbitrary time scale, $\left[\eta_{0}, \infty\right)_{\widehat{\pi}}=\left[\eta_{0}, \infty\right) \cap \widehat{\mathbb{T}},(\cdot)^{(\bar{\alpha})}$ is the conformable fractional dynamic operator of order $\bar{\alpha}(0<\bar{\alpha} \leq 1), \quad \varphi(\eta), a(\eta)$, $b(\eta) \in C_{r d}\left(\left[\eta_{0}, \infty\right)_{\widehat{\pi}},[0, \infty)\right)$, and $\mathbf{f} \in C(\mathbb{R}, \mathbb{R})$ such that $y f(y)>0$ and the function $h(\eta)=\left\{\begin{array}{ll}\leq 0, & {\left[\bar{\alpha}_{1}, \bar{\beta}_{1}\right],} \\ \geq 0, & {\left[\bar{\alpha}_{2}, \bar{\beta}_{2}\right]}\end{array}\right.$, where $\left[\bar{\alpha}_{i}, \bar{\beta}_{i}\right] \subset\left[\eta_{0}, \infty\right)_{\widehat{\pi}}$ is in $C_{r d}\left(\left[\eta_{0}, \infty\right)_{\widehat{\pi}},[0, \infty)\right)$. We constitute new interval criteria for oscillation of the solutions of equation (3) when the nonlinear function $\mathbf{f}$ is increasing and nonincreasing and extend the results to the Euler-type fractional dynamic equations.

By a solution of (3), we insist a nontrivial function $y(\eta) \in \mathbb{R}$ fulfilling (3) for $\eta \geq \eta_{0}$. If a solution of (3) is neither eventually positive (EP) nor eventually negative (EN), then it is called oscillatory. Or else, it is said to be nonoscillatory. If all solutions of (3) are oscillatory, then (3) is called oscillatory.

Before we proceed to the main results, we present essential preliminaries on conformable time-scale fractional calculus that will be used to justify further discussion. Terms and definitions are adopted from the papers $[2,18]$.

Definition 1 (see [2]). On any time scale $\widehat{\mathbb{T}}$, $\sigma(\eta)=\inf \{v \in \widehat{\mathbb{T}}, v>\eta\}$ and $\rho(\eta)=\sup \{v \in \widehat{\mathbb{T}}, v<\eta\}$ are defined as the forward and backward jump operators, respectively.

A point $\eta \in \widehat{\mathbb{T}}$ is known as right-dense if $\sigma(\eta)=\eta$, leftdense if $\rho(\eta)=\eta$, right-scattered if $\sigma(\eta)>\eta$, and left scattered if $\rho(\eta)<\eta$.
The graininess function $\mu(\eta)$ of the time scale is given by $\mu(\eta)=\sigma(\eta)-\eta \eta$

The set $\widehat{\mathbb{T}}^{k}=\{\widehat{\mathbb{T}} \backslash(\rho(\sup \widehat{\mathbb{T}}), \widehat{\mathbb{T}})$ if $\sup \widehat{\mathbb{\mathbb { V }}}<\infty \widehat{\mathbb{\mathbb { V }}}$ if $\widehat{\mathbb{T}}=\infty\}$.

Definition 2 (see [2]). A real-valued function $\mathbf{f}$ defined on $\widehat{\mathbb{T}}$ is known as rd-continuous if at all left-dense points, a finite left limit of $\mathbf{f}$ exists, and if it is continuous at each right-dense point.

Definition 3 (see [2]). A function $\zeta: \widehat{\mathbb{T}} \longrightarrow \mathbb{R}$ is known as regressive if $1+\mu(\eta) \zeta(\eta) \neq 0$ for $\eta \in \widehat{\mathbb{T}}^{k} . \mathscr{R}$ is the collection that consists of all rd-continuous regressive functions $\mathbf{f :} \widehat{\mathbb{T}} \longrightarrow \mathbb{R}$. We define $\mathscr{R}^{+}:=\{\mathbf{g} \in \mathscr{R}: 1+\mu(\eta) \mathbf{g}(\eta)>0$, $\eta \in \widehat{\mathbb{T}}\}$.

Definition 4 (see [2]). If $\zeta \in \mathscr{R}$, then the function defined by $e_{\zeta}(\eta, v)=\exp \left(\int_{v}^{\eta} \Theta_{\mu(v)}(\zeta(v)) \Delta v\right), \quad$ for $\eta \in \widehat{\mathbb{T}}, v \in \widehat{\mathbb{T}}^{k}$,

is called exponential, where $\Theta_{h}(z)=$ $\left\{\begin{array}{ll}\log (1+h z) / h, & h \neq 0, \\ z, & h=0\end{array}\right.$.

Also, $e_{\zeta}(\eta, v)$ is a nonzero real function and $e_{\zeta}\left(\sigma(\eta) ; \eta_{0}\right)=[1+\mu(\eta) \zeta(\eta)] e_{\zeta}\left(\eta, \eta_{0}\right)$

Definition 5 (see [18]). For $\eta \in \widehat{\mathbb{T}}^{k}, \bar{\alpha} \in(0,1]$ and g: $\widehat{\mathbb{T}} \longrightarrow \mathbb{R}$, the conformable fractional derivative of order $\bar{\alpha}$ for $\mathbf{g}$ at $\eta$ is $\mathbf{g}^{(\bar{\alpha})}(\eta)$ (if it exists) so that, for every positive $\epsilon$, there is a $\delta$-neighborhood $\mathfrak{N}$ fulfilling

$$
\begin{aligned}
& \left|[\mathbf{g}(\sigma(\eta))-\mathbf{g}(\nu)] \eta^{1-\bar{\alpha}}-\mathbf{g}^{(\bar{\alpha})}(\eta)(\sigma(\eta)-\nu)\right| \\
\leq & \epsilon|\sigma(\eta)-\nu|, \quad \text { for all } \nu \in \mathfrak{N} .
\end{aligned}
$$

Definition 6 (see [18]). If $\mathbf{G}^{(\bar{\alpha})}(\eta)=\mathbf{g}(\eta), \eta \in \widehat{\mathbb{\pi}}^{k}$, then $\mathbf{G}$ is said to be an $\bar{\alpha}$-order antiderivative of $\mathbf{g}$ and the integral of $\mathbf{g}$ given by

$$
\int_{a}^{b} \mathbf{g}(\eta) \Delta^{\bar{\alpha}} \eta=\int_{a}^{b} \mathbf{g}(\eta) \eta^{\bar{\alpha}-1} \Delta \eta=\mathbf{G}(b)-\mathbf{G}(a), \quad \text { where } a, b \in \widehat{\mathbb{T}},
$$

is named as Cauchy $\bar{\alpha}$-fractional.

Theorem 1 (see [18]). By the definition of $\bar{\alpha}$ - order conformable fractional derivative, $\mathbf{f}^{(\bar{\alpha})}(\eta)=\eta^{1-\bar{\alpha}} \mathbf{f}^{\Delta}(\eta)$. Also, $\mathbf{f}$ is decreasing (increasing) for $\eta>0$, if $\mathbf{f}^{(\bar{\alpha})}(\eta)<0(>0)$ for $\eta>0$.

Theorem 2 (see [19]). Let $\widetilde{\zeta}(\eta)=\eta^{\bar{\alpha}-1} \zeta(\eta), \bar{\alpha} \in(0,1]$. If $\widetilde{\zeta} \in \mathscr{R}$, then, for the IVP $x^{(\bar{\alpha})}(\eta)=\zeta(\eta) x(\eta)$ and $x\left(\eta_{0}\right)=1$ on $\widehat{\mathbb{T}}$, the unique solution is the exponential function $e_{\zeta}^{\sim}\left(\eta, \eta_{0}\right)$ for fixed $\eta_{0} \in \widehat{\mathbb{T}}$.

Theorem 3 (see [18]). Let $\mathbf{g}$, f be real-valued $\bar{\alpha}$ differentiable functions defined on $\widehat{\mathbb{T}}$ at a point $v$ in $\widehat{\mathbb{T}}^{k}$. Then,

$$
\begin{aligned}
& \text { (i) }(\mathbf{g f})^{(\bar{\alpha})}(v)=\mathbf{g}^{(\bar{\alpha})}(v) \mathbf{f}(v)+\mathbf{g}(\sigma(v)) \mathbf{f}^{(\bar{\alpha})}(v)=\mathbf{g}(v) \\
& \mathbf{f}^{(\bar{\alpha})}(v)+\mathbf{g}^{(\bar{\alpha})}(v) \mathbf{f}(\sigma(v))
\end{aligned}
$$


(ii) $(\mathbf{g} / \mathbf{f})^{(\bar{\alpha})}(v)=\left(\mathbf{g}^{(\bar{\alpha})}(v) \mathbf{f}(v)-\mathbf{g}(v) \mathbf{f}^{(\bar{\alpha})}(v) / \mathbf{f}(v) \mathbf{f}(\sigma\right.$ $(v))$ ), provided $\mathbf{f}(v) \mathbf{f}(\sigma(v)) \neq 0$

(iii) $\mathbf{g}(\sigma(v))=\mathbf{g}(v)+\mu(v) v^{1-\bar{\alpha}} \mathbf{g}^{(\bar{\alpha})}(v)$

Theorem 4 (see [18]). Assume $\bar{\alpha} \in(0,1] ; \mathbf{g}, \mathbf{f}: \widehat{\mathbb{T}} \longrightarrow \mathbb{R}$ be rd-continuous. Then,

$$
\int_{a}^{b} \mathbf{g}(\eta) \mathbf{f}^{(\bar{\alpha})}(\eta) \Delta^{\bar{\alpha}} \eta=[\mathbf{g}(\eta) \mathbf{f}(\eta)]_{a}^{b}-\int_{a}^{b} \mathbf{g}^{(\bar{\alpha})}(\eta) \mathbf{f}(\sigma(\eta)) \Delta^{\bar{\alpha}} \eta
$$

for $a, b \in \widehat{\mathbb{T}}$.

Definition 7. Assume $\mathbb{D}=\left\{(\eta, v) \mid \eta \geq v \geq \eta_{0}\right\}$. Then, class $\mathscr{G}$ is a collection of functions $\mathbf{G} \in C_{r d}(\mathbb{D}, \mathbb{R})$ so that $\mathbf{G}(\eta, \eta)=$ 0 for $\eta \geq \eta_{0} ; \mathbf{G}(\eta, v)>0$ for $\eta>v \geq \eta_{0}$ and $\mathbf{G}$ has continuous $\bar{\alpha}$-partial fractional derivatives $\mathbf{G}^{(\bar{\alpha})_{\eta}}(\eta, v)$ and $\mathbf{G}^{(\bar{\alpha})_{v}}(\eta, v)$ with respect to $\eta$ and $v$, respectively, such that $\mathbf{G}^{(\bar{\alpha})_{\eta}}(\eta, v)=$ $h_{1}(\eta, v) \sqrt{\mathbf{G}(\eta, v)} \quad$ and $\quad \mathbf{G}^{(\bar{\alpha})_{v}}(\eta, v)=-h_{2}(\eta, v) \sqrt{\mathbf{G}(\eta, v)}$, where $h_{1}, h_{2} \in L_{\text {loc }}\left(\mathbb{D},[0, \infty)_{\widehat{\mathbb{T}}}\right)$.

For simplicity, we use the notion as follows:

$$
\Phi(\eta)=-\frac{\eta^{\bar{\alpha}-1} a(\eta)}{\varphi(\eta)}
$$

\section{Main Results}

This part supplies the main theorems of the work. We will present the results in two folds based on the monotonicity of f and extend the results for fractional Euler-type dynamic equation.

Lemma 1. Suppose that $\Phi(\eta) \in \mathscr{R}^{+}$and $\int_{\eta_{0}}^{\infty} \Delta^{\bar{\alpha}} v / \varphi(v)=\infty$ hold.

(i) If (3) has an EP solution y, then there is a sufficiently large $\eta^{*} \in\left[\eta_{0}, \infty\right)_{\widehat{\pi}}$ so that

$$
\left(\frac{\varphi(\eta) y^{(\bar{\alpha})}(\eta)}{e_{\Phi(\eta)}\left(\eta, \eta_{0}\right)}\right)^{(\bar{\alpha})}<0, \quad y^{(\bar{\alpha})}(\eta)>0 \text { on the interval }\left[\eta^{*}, \infty\right)_{\widehat{\pi}} .
$$

(ii) If (3) has an $\mathbf{E N}$ solution $y$, then there is a sufficiently large $\eta^{*} \in\left[\eta_{0}, \infty\right)_{\widehat{\pi}}$ so that

$$
\left(\frac{\varphi(\eta) y^{(\bar{\alpha})}(\eta)}{e_{\Phi(\eta)}\left(\eta, \eta_{0}\right)}\right)^{(\bar{\alpha})}>0, \quad y^{(\bar{\alpha})}(\eta)<0 \text { on the interval }\left[\eta^{*}, \infty\right)_{\widehat{\mathbb{T}}} .
$$

Proof

(i) Since $y$ is $\mathbf{E P}$, there is an $\eta_{1}$ such that $y(\eta)>0$ on $\left[\eta_{1}, \infty\right)_{\widehat{\pi}}$. Now, we have

$$
\begin{aligned}
\left(\frac{\varphi(\eta) y^{(\bar{\alpha})}(\eta)}{e_{\Phi(\eta)}\left(\eta, \eta_{0}\right)}\right)^{(\bar{\alpha})} & =\frac{e_{\Phi(\eta)}\left(\eta, \eta_{0}\right)\left(\varphi(\eta) y^{(\bar{\alpha})}(\eta)\right)^{(\bar{\alpha})}-\varphi(\eta) y^{(\bar{\alpha})}(\eta)\left(e_{\Phi(\eta)}\left(\eta, \eta_{0}\right)\right)^{(\bar{\alpha})}}{e_{\Phi(\eta)}\left(\eta, \eta_{0}\right) e_{\Phi(\eta)}\left(\sigma(\eta), \eta_{0}\right)} \\
& =\frac{e_{\Phi(\eta)}\left(\eta, \eta_{0}\right)\left(\varphi(\eta) y^{(\bar{\alpha})}(\eta)\right)^{(\bar{\alpha})}+a(\eta) y^{(\bar{\alpha})}(\eta) e_{\Phi(\eta)}\left(\eta, \eta_{0}\right)}{e_{\Phi(\eta)}\left(\eta, \eta_{0}\right) e_{\Phi(\eta)}\left(\sigma(\eta), \eta_{0}\right)}=\frac{-b(\eta) \mathbf{f}(y(\eta))+h(\eta)}{e_{\Phi(\eta)}\left(\sigma(\eta), \eta_{0}\right)}
\end{aligned}
$$

By the assumptions, we take $\bar{\alpha}_{1}, \bar{\beta}_{1} \geq \eta_{1}$ such that $h(\eta) \leq 0$ on $\left[\bar{\alpha}_{1}, \bar{\beta}_{1}\right]_{\widehat{\pi}}$ with $\bar{\alpha}_{1}<\bar{\beta}_{1}$ and $y(\eta)>0$. Then, on the interval $\left[\bar{\alpha}_{1}, \bar{\beta}_{1}\right]_{\widehat{\pi}}$, we have

$\left(\frac{\varphi(\eta) y^{(\bar{\alpha})}(\eta)}{e_{\Phi(\eta)}\left(\eta, \eta_{0}\right)}\right)^{(\bar{\alpha})}=\frac{-b(\eta) \mathbf{f}(y(\eta))+h(\eta)}{e_{\Phi(\eta)}\left(\sigma(\eta), \eta_{0}\right)}<0$.

Since $\Phi \in \mathscr{R}^{+}$, we acquire that $e_{\Phi(\eta)}\left(\sigma(\eta), \eta_{0}\right)>0$ and $\varphi(\eta) y^{(\bar{\alpha})}(\eta) / e_{\Phi(\eta)}\left(\eta, \eta_{0}\right)$ is decreasing on $\left[\eta_{1}, \infty\right) \hat{\text {. }}$. Therefore, by the assumption that $\varphi$ is positive and $\Phi \in \mathscr{R}^{+}$, we get that $y^{(\bar{\alpha})}(\eta)$ is either negative or positive.

Assume that $y^{(\bar{\alpha})}<0$ on $\left[\eta_{2}, \infty\right)_{\widehat{\pi}}$ for sufficiently large $\eta_{2}>\eta_{1}$. Thus,

$$
\begin{aligned}
y(\eta)-y\left(\eta_{2}\right) & =\int_{\eta_{2}}^{\eta} \frac{\varphi(v) y^{(\bar{\alpha})}(v)}{\varphi(v)} \Delta^{\bar{\alpha}} v \\
& \leq \varphi\left(\eta_{2}\right) y^{(\bar{\alpha})}\left(\eta_{2}\right) \int_{\eta_{2}}^{\eta} \frac{\Delta^{\bar{\alpha}} v}{\varphi(v)}
\end{aligned}
$$

Letting $\eta \longrightarrow \infty$, we get $\lim _{\eta \longrightarrow \infty} y(\eta)=-\infty$. This leads to a contradiction that $y(\eta)$ is EP. Therefore, $y^{(\bar{\alpha})}(\eta)$ is $\mathbf{E P}$.

(ii) Since $y$ is $\mathbf{E N}$, there is a sufficiently large $\eta_{1}$ so that $y(\eta)>0$ on $\left[\eta_{0}, \infty\right)_{\hat{\mathbb{T}}}$. Now, we have 


$$
\begin{aligned}
\left(\frac{\varphi(\eta) y^{(\bar{\alpha})}(\eta)}{e_{\Phi(\eta)}\left(\eta, \eta_{0}\right)}\right)^{(\bar{\alpha})} & =\frac{e_{\Phi(\eta)}\left(\eta, \eta_{0}\right)\left(\varphi(\eta) y^{(\bar{\alpha})}(\eta)\right)^{(\bar{\alpha})}-\varphi(\eta) y^{(\bar{\alpha})}(\eta)\left(e_{\Phi(\eta)}\left(\eta, \eta_{0}\right)\right)^{(\bar{\alpha})}}{e_{\Phi(\eta)}\left(\eta, \eta_{0}\right) e_{\Phi(\eta)}\left(\sigma(\eta), \eta_{0}\right)} \\
& =\frac{e_{\Phi(\eta)}\left(\eta, \eta_{0}\right)\left(\varphi(\eta) y^{(\bar{\alpha})}(\eta)\right)^{(\bar{\alpha})}+a(\eta) y^{(\bar{\alpha})}(\eta) e_{\Phi(\eta)}\left(\eta, \eta_{0}\right)}{e_{\Phi(\eta)}\left(\eta, \eta_{0}\right) e_{\Phi(\eta)}\left(\sigma(\eta), \eta_{0}\right)} \\
& =\frac{-b(\eta) \mathbf{f}(y(\eta))+h(\eta)}{e_{\Phi(\eta)}\left(\sigma(\eta), \eta_{0}\right)} .
\end{aligned}
$$

By the assumptions, we can choose $\bar{\alpha}_{2}, \bar{\beta}_{2} \geq \eta_{1}$ such that $h(\eta) \geq 0$ on $\left[\bar{\alpha}_{2}, \bar{\beta}_{2}\right]_{\widehat{\pi}}$ with $\bar{\alpha}_{2}<\bar{\beta}_{2}$ and $y(\eta)<0$. Then, on $\left[\bar{\alpha}_{2}, \bar{\beta}_{2}\right]_{\widehat{\mathbb{T}}}$, we attain

$$
\left(\frac{\varphi(\eta) y^{(\bar{\alpha})}(\eta)}{e_{\Phi(\eta)}\left(\eta, \eta_{0}\right)}\right)^{(\bar{\alpha})}=\frac{-b(\eta) \mathbf{f}(y(\eta))+h(\eta)}{e_{\Phi(\eta)}\left(\sigma(\eta), \eta_{0}\right)}>0 .
$$

Since $\Phi \in \mathscr{R}^{+}$, we get that $e_{\Phi(\eta)}\left(\sigma(\eta), \eta_{0}\right)>0$ and $\varphi(\eta) y^{(\bar{\alpha})}(\eta) / e_{\Phi(\eta)}\left(\eta, \eta_{0}\right)$ is increasing on $\left[\eta_{1}, \infty\right) \widehat{\pi}$. Therefore, by the assumption of $\varphi(\eta)>0$ and $\Phi(\eta) \in \mathscr{R}^{+}$, we get that $y^{(\bar{\alpha})}(\eta)$ is either negative or positive.

Assume that $y^{(\bar{\alpha})}(\eta)>0$ on $\left[\eta_{2}, \infty\right)$ for sufficiently large $\eta_{2}>\eta_{1}$. Therefore,

$$
\begin{aligned}
y(\eta)-y\left(\eta_{2}\right) & =\int_{\eta_{2}}^{\eta} \frac{\varphi(v) y^{(\bar{\alpha})}(v)}{\varphi(v)} \Delta^{\bar{\alpha}} v \\
& \geq \varphi\left(\eta_{2}\right) y^{(\bar{\alpha})}\left(\eta_{2}\right) \int_{\eta_{2}}^{\eta} \frac{\Delta^{\bar{\alpha}} v}{\varphi(v)} .
\end{aligned}
$$

Letting $\eta \longrightarrow \infty$, we get $\lim _{\eta \longrightarrow \infty} y(\eta)=\infty$, and this leads to a contradiction that $y(\eta)$ is EN. Therefore, $y^{(\bar{\alpha})}$ is $\mathbf{E N}$.

3.1. Oscillation Criteria for (3) When f Is Not Necessarily Increasing. To establish oscillation criteria, we make use of the following assumption:

$$
\left(H_{1}\right) \mathbf{f}(y) / y \geq \mathbf{K} \forall y \neq 0 \text { and for some } \mathbf{K}>0 .
$$

Theorem 5. Let $\Phi(\eta) \in \mathscr{R}^{+}$. If $\int_{\eta_{0}}^{\infty} \Delta^{\bar{\alpha}} v / \varphi(v)=\infty$ and $\left(H_{1}\right)$ holds. If there exists $\mathbf{c}_{i} \in\left(\bar{\alpha}_{i}, \bar{\beta}_{i}\right), i=1,2$, a function $\Theta(\eta)>0$ on $[T, \infty)_{\hat{\mathbb{T}}}$ and $\mathbf{G} \in \mathscr{G}$ for sufficiently large $T$ such that

$$
\begin{aligned}
& \frac{1}{\mathbf{G}\left(\mathbf{c}_{i}, \bar{\alpha}_{i}\right)} \int_{\bar{\alpha}_{i}}^{\mathbf{c}_{i}} \mathbf{G}\left(v, \bar{\alpha}_{i}\right)\left\{\frac{\mathbf{K} b(v) \Theta(v)}{e_{\Phi(v)}\left(\sigma(v), \eta_{0}\right)}-\frac{\varphi(v)\left[z^{(\bar{\alpha})}(v) \sqrt{\mathbf{G}\left(v, \bar{\alpha}_{i}\right)}+h_{1}\left(v, \bar{\alpha}_{i}\right) \Theta(\sigma(v))\right]^{2}}{4 \mathbf{G}\left(v, \bar{\alpha}_{i}\right) \Theta(v) e_{\Phi(v)}\left(v, \eta_{0}\right)}\right\} \Delta^{\bar{\alpha}} v \\
& +\frac{1}{\mathbf{G}\left(\bar{\beta}_{i}, \mathbf{c}_{i}\right)} \int_{\mathbf{c}_{i}}^{\bar{\beta}_{i}} \mathbf{G}\left(\bar{\beta}_{i}, v\right)\left\{\frac{\mathbf{K} b(v) \Theta(v)}{e_{\Phi(v)}\left(\sigma(v), \eta_{0}\right)}-\frac{\varphi(v)\left[z^{(\bar{\alpha})}(v) \sqrt{\mathbf{G}\left(\bar{\beta}_{i}, v\right)}-h_{2}\left(\bar{\beta}_{i}, v\right) \Theta(\sigma(v))\right]^{2}}{4 \mathbf{G}\left(\bar{\beta}_{i}, v\right) \Theta(v) e_{\Phi(v)}\left(v, \eta_{0}\right)}\right\} \Delta^{\bar{\alpha}} v>0
\end{aligned}
$$

then (3) is oscillatory.

Proof. Assume that (3) has a nonoscillatory solution $y$.

$$
w(\eta)=\frac{\Theta(\eta) \varphi(\eta) y^{(\bar{\alpha})}(\eta)}{y(\eta) e_{\Phi(\eta)}\left(\eta, \eta_{0}\right)}
$$

Then, $y$ is either EN or EP for $\eta \geq \eta_{1} \geq \eta_{0}$. Define a generalized Riccati function as follows:
By Lemma 1 , clearly $w(\eta) \geq 0$ and 


$$
\begin{aligned}
w^{(\bar{\alpha})}(\eta)= & \left.\frac{\Theta}{y}\right)(\eta)\left[\frac{e_{\Phi(\eta)}\left(\eta, \eta_{0}\right)\left[\varphi(\eta) y^{(\bar{\alpha})}(\eta)\right]^{(\bar{\alpha})}+a(\eta) y^{(\bar{\alpha})}(\eta) e_{\Phi(\eta)}\left(\eta, \eta_{0}\right)}{e_{\Phi(\eta)}\left(\eta, \eta_{0}\right) e_{\Phi(\eta)}\left(\sigma(\eta), \eta_{0}\right)}\right] \\
& +\left[\frac{y(\eta) z^{(\bar{\alpha})}(\eta)-\Theta(\eta) y^{(\bar{\alpha})}(\eta)}{y(\sigma(\eta)) y(\eta)}\right] \frac{\varphi(\sigma(\eta)) y^{(\bar{\alpha})}(\sigma(\eta))}{e_{\Phi(\eta)}\left(\sigma(\eta), \eta_{0}\right)} \\
& \cdot \frac{\Theta(\eta)}{y(\eta)}\left[\frac{-b(\eta) \mathbf{f}(y(\eta))+h(\eta)}{e_{\Phi(\eta)}\left(\sigma(\eta), \eta_{0}\right)}\right]+z^{(\bar{\alpha})}(\eta)\left(\frac{w}{\Theta}\right)(\sigma(\eta)) \\
& -\frac{\Theta(\eta) \varphi(\sigma(\eta)) y^{(\bar{\alpha})}(\eta) y^{(\bar{\alpha})}(\sigma(\eta))}{y(\sigma(\eta)) y(\eta) e_{\Phi(\eta)}\left(\sigma(\eta), \eta_{0}\right)} \\
\leq & \frac{-\mathbf{K} b(\eta) \Theta(\eta)}{e_{\Phi(\eta)}\left(\sigma(\eta), \eta_{0}\right)}+\frac{\Theta(\eta) h(\eta)}{y(\eta) e_{\Phi(\eta)}\left(\sigma(\eta), \eta_{0}\right)}+z^{(\bar{\alpha})}(\eta)\left(\frac{w}{\Theta}\right)(\sigma(\eta)) \\
& -\frac{\Theta(\eta) y^{(\bar{\alpha})}(\eta) \varphi(\sigma(\eta)) y^{(\bar{\alpha})}(\sigma(\eta))}{y(\eta) y(\sigma(\eta)) e_{\Phi(\eta)}\left(\sigma(\eta), \eta_{0}\right)} .
\end{aligned}
$$

By assumptions, we take $\bar{\alpha}_{i}, \bar{\beta}_{i} \geq \eta_{1}$ for $i=1,2$ so that $h(\eta) \leq 0$ on $\left[\bar{\alpha}_{1}, \bar{\beta}_{1}\right]$ with $\bar{\alpha}_{1}<\bar{\beta}_{1}$ and $y(\eta)$ is $\mathbf{E P}$, or $h(\eta) \geq 0$ on $\left[\bar{\alpha}_{2}, \bar{\beta}_{2}\right]$ with $\bar{\alpha}_{2}<\bar{\beta}_{2}$ and $y(\eta)$ is EN. On the intervals $\left[\bar{\alpha}_{1}, \bar{\beta}_{1}\right]$ and $\left[\bar{\alpha}_{2}, \bar{\beta}_{2}\right]$, the above inequality implies that

$$
w^{(\bar{\alpha})}(\eta) \leq \frac{-\mathbf{K} b(\eta) \Theta(\eta)}{e_{\Phi(\eta)}\left(\sigma(\eta), \eta_{0}\right)}+z^{(\bar{\alpha})}(\eta)\left(\frac{w}{\Theta}\right)(\sigma(\eta))-\frac{\Theta(\eta) e_{\Phi(\eta)}\left(\eta, \eta_{0}\right)}{\varphi(\eta)}\left[\left(\frac{w}{\Theta}\right)(\sigma(\eta))\right]^{2}
$$

By multiplying $\mathbf{G}(\eta, v)$ and taking $\bar{\alpha}$ - fractional integral for (20) from $c_{i}$ to $\eta$, we have

$$
\begin{aligned}
& \int_{\mathbf{c}_{i}}^{\eta} \frac{\mathbf{K} b(v) \Theta(v)}{e_{\Phi(v)}\left(\sigma(v), \eta_{0}\right)} \mathbf{G}(\eta, v) \Delta^{\bar{\alpha}} v \\
& \leq-\int_{\mathbf{c}_{i}}^{\eta} w^{(\bar{\alpha})}(v) \mathbf{G}(\eta, v) \Delta^{\bar{\alpha}} v+\int_{\mathbf{c}_{i}}^{\eta} z^{(\bar{\alpha})}(\eta)\left(\frac{w}{\Theta}\right)(\sigma(\eta)) \mathbf{G}(\eta, v) \Delta^{\bar{\alpha}} v \\
& -\int_{\mathbf{c}_{i}}^{\eta} \frac{\Theta(v) e_{\Phi(v)}\left(v, \eta_{0}\right)}{\varphi(v)}\left[\left(\frac{w}{\Theta}\right)(\sigma(\eta))\right]^{2} \mathbf{G}(\eta, v) \Delta^{\bar{\alpha}} v \leq \mathbf{G}\left(\eta, \mathbf{c}_{i}\right) w\left(\mathbf{c}_{i}\right) \\
& +\int_{\mathbf{c}_{i}}^{\eta} w(\sigma(v)) \mathbf{G}^{(\bar{\alpha})}(\eta, v) \Delta^{\bar{\alpha}} v+\int_{\mathbf{c}_{i}}^{\eta} \frac{z^{(\bar{\alpha})}(v) w(\sigma(v)) \mathbf{G}(\eta, v)}{\Theta(\sigma(v))} \Delta^{\bar{\alpha}} v \\
& -\int_{\mathbf{c}_{i}}^{\eta} \frac{\Theta(v) e_{\Phi(v)}\left(v, \eta_{0}\right)}{\varphi(v)}\left[\left(\frac{w}{\Theta}\right)(\sigma(v))\right]^{2} \mathbf{G}(\eta, v) \Delta^{\bar{\alpha}} v \\
& \leq \mathbf{G}\left(\eta, \mathbf{c}_{i}\right) w\left(\mathbf{c}_{i}\right)+\int_{c_{i}}^{\eta} \mathbf{G}(\eta, v)\left[z^{(\bar{\alpha})}(v)-\frac{h_{2}(\eta, v) \Theta(\sigma(v))}{\sqrt{\mathbf{G}(\eta, v)}}\right] \frac{w(\sigma(v))}{\Theta(\sigma(v))} \Delta^{\bar{\alpha}} v \\
& -\int_{\mathbf{c}_{i}}^{\eta} \mathbf{G}(\eta, v) \frac{\Theta(v) e_{\Phi(v)}\left(v, \eta_{0}\right)}{\varphi(v)}\left[\frac{w(\sigma(v))}{\Theta(\sigma(v))}\right]^{2} \Delta^{\bar{\alpha}} v \leq \mathbf{G}\left(\eta, \mathbf{c}_{i}\right) w\left(\mathbf{c}_{i}\right) \\
& d+\int_{\mathbf{c}_{i}}^{\eta} \mathbf{G}(\eta, v) \frac{\varphi(v)\left[z^{(\bar{\alpha})}(v) \sqrt{\mathbf{G}(\eta, v)}-\Theta(\sigma(v)) h_{2}(\eta, v)\right]^{2}}{4 \mathbf{G}(\eta, v) \Theta(v) e_{\Phi(v)}\left(v, \eta_{0}\right)} \Delta^{\bar{\alpha}} v
\end{aligned}
$$


Letting $\eta \longrightarrow \bar{\beta}_{i}^{-}$, we get

$$
\begin{aligned}
& \int_{c_{i}}^{\bar{\beta}_{i}} \mathbf{G}(\eta, v)\left\{\frac{\mathbf{K} b(\eta) \Theta(\eta)}{e_{\Phi(\eta)}\left(\sigma(\eta), \eta_{0}\right)}-\frac{\varphi(v)\left[z^{(\bar{\alpha})}(v) \sqrt{\mathbf{G}(\eta, v)}-\Theta(\sigma(v)) h_{2}(\eta, v)\right]^{2}}{4 \mathbf{G}(\eta, v) \Theta(v) e_{\Phi(v)}\left(v, \eta_{0}\right)}\right\} \Delta^{\bar{\alpha}} v \\
& \leq \mathbf{G}\left(\bar{\beta}_{i}, \mathbf{c}_{i}\right) w\left(\mathbf{c}_{i}\right) .
\end{aligned}
$$

By multiplying $\mathbf{G}(v, \eta)$ and taking integration of $\bar{\alpha}$-order from $\eta$ to $\mathbf{c}_{i}$ for (20), we have

$$
\begin{aligned}
& \int_{\eta}^{\mathbf{c}_{i}} \mathbf{G}(v, \eta) \frac{\mathbf{K} b(v) \Theta(v)}{e_{\Phi(v)}\left(\sigma(v), \eta_{0}\right)} \Delta^{\bar{\alpha}} v \\
\leq & -\int_{\eta}^{\mathbf{c}_{i}} \mathbf{G}(v, \eta) w^{(\bar{\alpha})}(v) \Delta^{\bar{\alpha}} v+\int_{\eta}^{\mathbf{c}_{i}} \mathbf{G}(v, \eta) \frac{z^{(\bar{\alpha})}(v) w(\sigma(v))}{\Theta(\sigma(v))} \Delta^{\bar{\alpha}} v \\
& -\int_{\eta}^{\mathbf{c}_{i}} \mathbf{G}(v, \eta) \frac{\Theta(v) e_{\Phi(v)}\left(v, \eta_{0}\right)}{\varphi(v)}\left[\frac{w(\sigma(v))}{\Theta(\sigma(v))}\right]^{2} \Delta^{\bar{\alpha}} v \\
\leq & -\mathbf{G}\left(\mathbf{c}_{i}, \eta\right) w\left(\mathbf{c}_{i}\right)+\int_{\eta}^{\mathbf{c}_{i}} \mathbf{G}^{(\bar{\alpha})}(v, \eta) w(\sigma(v)) \Delta^{\bar{\alpha}} v+\int_{\eta}^{\mathbf{c}_{i}} \frac{\mathbf{G}(v, \eta) z^{(\bar{\alpha})}(v) w(\sigma(v))}{\Theta(\sigma(v))} \Delta^{\bar{\alpha}} v \\
& -\int_{\eta}^{\mathbf{c}_{i}} \mathbf{G}(v, \eta) \frac{\Theta(v) e_{\Phi(v)}\left(v, \eta_{0}\right)}{\varphi(v)}\left[\frac{w(\sigma(v))}{\Theta(\sigma(v))}\right]^{2} \Delta^{\bar{\alpha}} v \\
\leq & -\mathbf{G}\left(\mathbf{c}_{i}, \eta\right) w\left(\mathbf{c}_{i}\right)+\int_{\eta}^{\mathbf{c}_{i}} \mathbf{G}(v, \eta)\left[z^{(\bar{\alpha})}(v)+\frac{h_{1}(v, \eta) \Theta(\sigma(v))}{\sqrt{\mathbf{G}(\eta, v)}}\right] \frac{w(\sigma(v))}{\Theta(\sigma(v))} \Delta^{\bar{\alpha}} v \\
& -\int_{\eta}^{\mathbf{c}_{i}} \mathbf{G}(v, \eta) \frac{\Theta(v) e_{\Phi(v)}\left(v, \eta_{0}\right)}{\varphi(v)}\left[\frac{w(\sigma(v))}{\Theta(\sigma(v))}\right]^{2} \Delta^{\bar{\alpha}} v \\
\leq & -\mathbf{G}\left(\mathbf{c}_{i}, \eta\right) w\left(\mathbf{c}_{i}\right)+\int_{\eta}^{c_{i}} \mathbf{G}(v, \eta) \frac{\varphi(v)\left[z^{(\bar{\alpha})}(v) \sqrt{\mathbf{G}(v, \eta)}+h_{1}(v, \eta) \Theta(\sigma(v))\right]^{2}}{4 \mathbf{G}(v, \eta) \Theta(v) e_{\Phi(v)}\left(v, \eta_{0}\right)} \Delta^{\bar{\alpha}} v .
\end{aligned}
$$

Letting $\eta \longrightarrow \bar{\alpha}_{i}^{+}$, we get

$$
\begin{aligned}
& \int_{\bar{\alpha}_{i}}^{\mathbf{c}_{i}} \mathbf{G}(v, \eta)\left\{\frac{\mathbf{K} b(\eta) \Theta(\eta)}{e_{\Phi(\eta)}\left(\sigma(\eta), \eta_{0}\right)}-\frac{\varphi(v)\left[z^{(\bar{\alpha})}(v) \sqrt{\mathbf{G}(v, \eta)}+h_{1}(\eta, v) \Theta(\sigma(v))\right]^{2}}{4 \mathbf{G}(v, \eta) \Theta(v) e_{\Phi(v)}\left(v, \eta_{0}\right)}\right\} \Delta^{\bar{\alpha}} v \\
& \leq-\mathbf{G}\left(\mathbf{c}_{i}, \bar{\alpha}_{i}\right) w\left(\mathbf{c}_{i}\right) .
\end{aligned}
$$

Dividing (33) by $\mathbf{G}\left(\bar{\beta}_{i}, \mathbf{c}_{i}\right)$ and (24) by $\mathbf{G}\left(\mathbf{c}_{i}, \bar{\alpha}_{i}\right)$, we attain 


$$
\begin{aligned}
& \frac{1}{\mathbf{G}\left(\bar{\beta}_{i}, \mathbf{c}_{i}\right)} \int_{\mathbf{c}_{i}}^{\bar{\beta}_{i}} \mathbf{G}\left(\bar{\beta}_{i}, v\right)\left\{\frac{\mathbf{K} b(v) \Theta(v)}{e_{\Phi(v)}\left(\sigma(v), \eta_{0}\right)}-\frac{\varphi(v)\left[z^{(\bar{\alpha})}(v) \sqrt{\mathbf{G}\left(\bar{\beta}_{i}, v\right)}-h_{2}\left(\bar{\beta}_{i}, v\right) \Theta(\sigma(v))\right]^{2}}{4 \mathbf{G}\left(\bar{\beta}_{i}, v\right) \Theta(v) e_{\Phi(v)}\left(v, \eta_{0}\right)}\right\} \Delta^{\bar{\alpha}} v \leq w\left(\mathbf{c}_{i}\right), \\
& \frac{1}{\mathbf{G}\left(\mathbf{c}_{i}, \bar{\alpha}_{i}\right)} \int_{\bar{\alpha}_{i}}^{\mathbf{c}_{i}} \mathbf{G}\left(v, \bar{\alpha}_{i}\right)\left\{\frac{\mathbf{K} b(v) \Theta(v)}{e_{\Phi(v)}\left(\sigma(v), \eta_{0}\right)}-\frac{\varphi(v)\left[z^{(\bar{\alpha})}(v) \sqrt{\mathbf{G}\left(v, \bar{\alpha}_{i}\right)}+h_{1}\left(v, \bar{\alpha}_{i}\right) \Theta(\sigma(v))\right]^{2}}{4 \mathbf{G}\left(v, \bar{\alpha}_{i}\right) \Theta(v) e_{\Phi(v)}\left(v, \eta_{0}\right)}\right\} \Delta^{\bar{\alpha}} v \leq-w\left(\mathbf{c}_{i}\right) .
\end{aligned}
$$

Adding the above two inequalities, we get a contradiction to hypothesis.
Remark 1. Assume that the condition of Theorem 5 holds for every $T \geq 0$ and function $\mathbf{G}(v, \eta) \in \mathscr{G}$ for sufficiently large $T$. If there exist some $\mathbf{c}_{i} \in\left(\bar{\alpha}_{i}, \bar{\beta}_{i}\right), i=1,2$ so that

$$
\begin{gathered}
\int_{\bar{\alpha}_{i}}^{\mathbf{c}_{i}} \mathbf{G}\left(v, \bar{\alpha}_{i}\right)\left\{\frac{\mathbf{K} b(v) \Theta(v)}{e_{\Phi(v)}\left(\sigma(v), \eta_{0}\right)}-\frac{\varphi(v)\left[z^{(\bar{\alpha})}(v) \sqrt{\mathbf{G}\left(v, \bar{\alpha}_{i}\right)}+h_{1}\left(v, \bar{\alpha}_{i}\right) \Theta(\sigma(v))\right]^{2}}{4 \mathbf{G}\left(v, \bar{\alpha}_{i}\right) \Theta(v) e_{\Phi(v)}\left(v, \eta_{0}\right)}\right\} \Delta^{\bar{\alpha}} v>0, \\
\int_{\mathbf{c}_{i}}^{\bar{\beta}_{i}} \mathbf{G}\left(\bar{\beta}_{i}, v\right)\left\{\frac{\mathbf{K} b(v) \Theta(v)}{e_{\Phi(v)}\left(\sigma(v), \eta_{0}\right)}-\frac{\varphi(v)\left[z^{(\bar{\alpha})}(v) \sqrt{\mathbf{G}\left(\bar{\beta}_{i}, v\right)}-h_{2}\left(\bar{\beta}_{i}, v\right) \Theta(\sigma(v))\right]^{2}}{4 \mathbf{G}\left(\bar{\beta}_{i}, v\right) \Theta(v) e_{\Phi(v)}\left(v, \eta_{0}\right)}\right\} \Delta^{\bar{\alpha}} v>0,
\end{gathered}
$$

for $i=1,2$, then (3) is oscillatory.

Let $\mathbf{G}=\mathbf{G}(\eta-v) \in \mathscr{G}$. Then, $h_{1}(\eta-v)=h_{2}(\eta-v)$, and we use $h(\eta-v)$ for them. By using this $\mathbf{G}(\eta-v)$ in Theorem 5 , we have the corollary as follows.
Corollary 1. Suppose that all the assumptions of Theorem 5 hold and for every $T \geq \eta_{0}$, there is a $T \leq \bar{\alpha}_{1}<2 \mathbf{c}_{1}-\bar{\alpha}_{1} \leq \bar{\alpha}_{2}<$ $2 \mathbf{c}_{2}-\bar{\alpha}_{2}$ so that $h(\eta)= \begin{cases}\leq 0, & \eta \in\left[\bar{\alpha}_{1}, 2 \mathbf{c}_{1}-\bar{\alpha}_{1}\right], \\ \geq 0, & \eta \in\left[\bar{\alpha}_{2}, 2 \mathbf{c}_{2}-\bar{\alpha}_{2}\right] . \text { If there is }\end{cases}$ $a \mathbf{G}:=\mathbf{G}(\eta-v) \in \mathscr{G}$ such that

$$
\frac{1}{\mathbf{G}\left(\mathbf{c}_{i}-\bar{\alpha}_{i}\right)} \int_{\bar{\alpha}_{i}}^{\mathbf{c}_{i}} \mathbf{G}\left(v-\bar{\alpha}_{i}\right)\left\{\frac{2 \mathbf{K} b(v) \Theta(v)}{e_{\Phi(v)}\left(\sigma(v), \eta_{0}\right)}-\frac{\varphi(v)\left[\left(z^{(\bar{\alpha})}(v)\right)^{2} \mathbf{G}\left(v-\bar{\alpha}_{i}\right)+h^{2}\left(v-\bar{\alpha}_{i}\right) \Theta^{2}(\sigma(v))\right]}{2 \mathbf{G}\left(v-\bar{\alpha}_{i}\right) \Theta(v) e_{\Phi(v)}\left(v, \eta_{0}\right)}\right\} \Delta^{\bar{\alpha}} v>0
$$

then (3) is oscillatory.

3.2. Oscillation Criteria for (3) When $\mathbf{f}$ Is Increasing. To establish oscillation criteria, we make use of the following assumption:
(H2) $f^{\prime}$ exists and $f^{\prime}(y) \geq \mathbf{M}$ for all $y \neq 0$ and for some $\mathbf{M}>0$

Theorem 6. Let $\Phi(\eta) \in \mathscr{R}^{+}$. If $\left(H_{2}\right), \int_{\eta_{0}}^{\infty} \Delta^{\bar{\alpha}} v / \varphi(v)=\infty$ holds. If there exists $\mathbf{c}_{i} \in\left(\bar{\alpha}_{i}, \bar{\beta}_{i}\right), i=1,2$, a function $\Theta(\eta)>0$ on $[T, \infty)_{\widehat{\pi}}$ and $\mathbf{G} \in \mathscr{G}$ for sufficiently large $T$ such that

$$
\begin{aligned}
& \operatorname{frac} 1 \mathbf{G}\left(\mathbf{c}_{i}, \bar{\alpha}_{i}\right) \int_{\bar{\alpha}_{i}}^{\mathbf{c}_{i}} \mathbf{G}\left(v, \bar{\alpha}_{i}\right)\left\{\frac{b(v) \Theta(v)}{e_{\Phi(v)}\left(\sigma(v), \eta_{0}\right)}-\frac{\varphi(\eta)\left[z^{(\bar{\alpha})}(v) \sqrt{\mathbf{G}\left(v, \bar{\alpha}_{i}\right)}+h_{1}\left(v, \bar{\alpha}_{i}\right) \Theta(\sigma(v))\right]^{2}}{4 \mathbf{M G}\left(v, \bar{\alpha}_{i}\right) \Theta(v) e_{\Phi(v)}\left(v, \eta_{0}\right)}\right\} \Delta^{\bar{\alpha}} v \\
& +\frac{1}{\mathbf{G}\left(\bar{\beta}_{i}, \mathbf{c}_{i}\right)} \int_{\mathbf{c}_{i}}^{\bar{\beta}_{i}} \mathbf{G}\left(\bar{\beta}_{i}, v\right)\left\{\frac{b(v) \Theta(v)}{e_{\Phi(v)}\left(\sigma(v), \eta_{0}\right)}-\frac{\varphi(\eta)\left[z^{(\bar{\alpha})}(v) \sqrt{\mathbf{G}\left(\bar{\beta}_{i}, v\right)}-h_{2}\left(\bar{\beta}_{i}, v\right) \Theta(\sigma(v))\right]^{2}}{4 \mathbf{M G}\left(\bar{\beta}_{i}, v\right) \Theta(v) e_{\Phi(v)}\left(v, \eta_{0}\right)}\right\} \Delta^{\bar{\alpha}} v>0,
\end{aligned}
$$

then (3) is oscillatory. 
Proof. Assume that (3) has a nonoscillatory solution $y$. Define a generalized Riccati function as follows:

$$
w(\eta)=\frac{\Theta(\eta) \varphi(\eta) y^{(\bar{\alpha})}(\eta)}{\mathbf{f}(y(\eta)) e_{\Phi(\eta)}\left(\eta, \eta_{0}\right)} .
$$

$$
\begin{aligned}
w^{(\bar{\alpha})}(\eta)= & \frac{\Theta(\eta)}{\mathbf{f}(y(\eta))}\left[\frac{e_{\Phi(\eta)}\left(\eta, \eta_{0}\right)\left[\varphi(\eta) y^{(\bar{\alpha})}(\eta)\right]^{(\bar{\alpha})}+a(\eta) e_{\Phi(\eta)}\left(\eta, \eta_{0}\right) y^{(\bar{\alpha})}(\eta)}{e_{\Phi(\eta)}\left(\sigma(\eta), \eta_{0}\right) e_{\Phi(\eta)}\left(\eta, \eta_{0}\right)}\right] \\
& +\left[\frac{\mathbf{f}(y(\eta)) z^{(\bar{\alpha})}(\eta)-\Theta(\eta)(\mathbf{f}(y(\eta)))^{(\bar{\alpha})}}{\mathbf{f}(y(\sigma(\eta))) \mathbf{f}(y(\eta))}\right] \frac{\varphi(\sigma(\eta)) y^{(\bar{\alpha})}(\sigma(\eta))}{e_{\Phi(\eta)}\left(\sigma(\eta), \eta_{0}\right)} \\
= & \frac{\Theta(\eta)}{\mathbf{f}(y(\eta))}\left[\frac{-b(\eta) \mathbf{f}(y(\eta))+h(\eta)}{e_{\Phi(\eta)}\left(\sigma(\eta), \eta_{0}\right)}\right]+z^{(\bar{\alpha})}(\eta)\left(\frac{w}{\Theta}\right)(\sigma(\eta)) \\
& -\frac{\Theta(\eta) \varphi(\sigma(\eta))(\mathbf{f}(y(\eta))){ }^{(\bar{\alpha})} y^{(\bar{\alpha})}(\sigma(\eta))}{\mathbf{f}(y(\sigma(\eta))) \mathbf{f}(y(\eta)) e_{\Phi(\eta)}\left(\sigma(\eta), \eta_{0}\right)} \\
= & \frac{-b(\eta) \Theta(\eta)}{e_{\Phi(\eta)}\left(\sigma(\eta), \eta_{0}\right)}+\frac{h(\eta) \Theta(\eta)}{\mathbf{f}(y(\eta)) e_{\Phi(\eta)}\left(\sigma(\eta), \eta_{0}\right)}+z^{(\bar{\alpha})}(\eta)\left(\frac{w}{\Theta}\right)(\sigma(\eta)) \\
& -\frac{\Theta(\eta) \varphi(\sigma(\eta))(\mathbf{f}(y(\eta))))^{(\bar{\alpha})} y^{(\bar{\alpha})}(\sigma(\eta))}{\mathbf{f}(y(\sigma(\eta))) \mathbf{f}(y(\eta)) e_{\Phi(\eta)}\left(\sigma(\eta), \eta_{0}\right)} .
\end{aligned}
$$

By the hypothesis, we take $\bar{\alpha}_{i}, \bar{\beta}_{i} \geq \eta_{1}$ for $i=1,2$ such that $h(\eta) \leq 0$ on $\left[\bar{\alpha}_{1}, \bar{\beta}_{1}\right]$ with $\bar{\alpha}_{1}<\bar{\beta}_{1}$ and $y(\eta)$ is $\mathbf{E P}$, or $h(\eta) \geq 0$ on $\left[\bar{\alpha}_{2}, \bar{\beta}_{2}\right]$ with $\bar{\alpha}_{2}<\bar{\beta}_{2}$ and $y(\eta)$ is $\mathbf{E N}$. On the intervals $\left[\bar{\alpha}_{1}, \bar{\beta}_{1}\right]$ and $\left[\bar{\alpha}_{2}, \bar{\beta}_{2}\right]$, we have

$$
\begin{aligned}
w^{(\bar{\alpha})}(\eta) & \leq \frac{-b(\eta) \Theta(\eta)}{e_{\Phi(\eta)}\left(\sigma(\eta), \eta_{0}\right)}+z^{(\bar{\alpha})}(\eta)\left(\frac{w}{\Theta}\right)(\sigma(\eta))-\frac{\Theta(\eta) f^{\prime}(y(\eta)) y^{(\bar{\alpha})}(\eta) \varphi(\sigma(\eta)) y^{(\bar{\alpha})}(\sigma(\eta))}{\mathbf{f}(y(\eta)) \mathbf{f}(y(\sigma(\eta))) e_{\Phi(\eta)}\left(\sigma(\eta), \eta_{0}\right)} \\
& \leq \frac{-b(\eta) \Theta(\eta)}{e_{\Phi(\eta)}\left(\sigma(\eta), \eta_{0}\right)}+z^{(\bar{\alpha})}(\eta)\left(\frac{w}{\Theta}\right)(\sigma(\eta))-\frac{\mathbf{M}(\eta) e_{\Phi(\eta)}\left(\eta, \eta_{0}\right)}{\varphi(\eta)}\left(\frac{w}{z}\right)^{2}(\sigma(\eta)) .
\end{aligned}
$$

By multiplying $\mathbf{G}(\eta, v)$ and taking $\bar{\alpha}$ - fractional integral for (31) from $\mathbf{c}_{i}$ to $\eta$, we have

$$
\begin{aligned}
& \int_{\mathbf{c}_{i}}^{\eta} \frac{b(v) \Theta(v)}{e_{\Phi(v)}\left(\sigma(v), \eta_{0}\right)} \mathbf{G}(\eta, v) \Delta^{\bar{\alpha}} v \leq-\int_{\mathbf{c}_{i}}^{\eta} w^{(\bar{\alpha})}(v) \mathbf{G}(\eta, v) \Delta^{\bar{\alpha}} v \\
& \quad+\int_{\mathbf{c}_{i}}^{\eta} \frac{z^{(\bar{\alpha})}(v) w(\sigma(v))}{\Theta(\sigma(v))} \mathbf{G}(\eta, v) \Delta^{\bar{\alpha}} v-\int_{\mathbf{c}_{i}}^{\eta} \frac{\mathbf{M} \Theta(v) e_{\Phi(v)}\left(v, \eta_{0}\right)}{\varphi(v)}\left[\frac{w(\sigma(v))}{\Theta(\sigma(v))}\right]^{2} \mathbf{G}(\eta, v) \Delta^{\bar{\alpha}} v \\
& \leq \mathbf{G}\left(\eta, \mathbf{c}_{i}\right) w\left(\mathbf{c}_{i}\right)+\int_{\mathbf{c}_{i}}^{\eta} w(\sigma(v)) \mathbf{G}^{(\bar{\alpha})}(\eta, v) \Delta^{\bar{\alpha}} v+\int_{\mathbf{c}_{i}}^{\eta} \frac{z^{(\bar{\alpha})}(v) w(\sigma(v)) \mathbf{G}(\eta, v)}{\Theta(\sigma(v))} \Delta^{\bar{\alpha}} v
\end{aligned}
$$




$$
\begin{aligned}
&-\int_{\mathbf{c}_{i}}^{\eta} \frac{\mathbf{M} \Theta(v) e_{\Phi(v)}\left(v, \eta_{0}\right)}{\varphi(v)}\left[\frac{w(\sigma(v))}{\Theta(\sigma(v))}\right]^{2} \mathbf{G}(\eta, v) \Delta^{\bar{\alpha}} v \leq \mathbf{G}\left(\eta, \mathbf{c}_{i}\right) w\left(\mathbf{c}_{i}\right) \\
&+\int_{\mathbf{c}_{i}}^{\eta} \mathbf{G}(\eta, v)\left[z^{(\bar{\alpha})}(v)-\frac{h_{2}(\eta, v) \Theta(\sigma(v))}{\sqrt{\mathbf{G}(\eta, v)}}\right] \frac{w(\sigma(v))}{\Theta(\sigma(v))} \Delta^{\bar{\alpha}} v \\
&-\int_{\mathbf{c}_{i}}^{\eta} \mathbf{G}(\eta, v) \frac{\mathbf{M} \Theta(v) e_{\Phi(v)}\left(v, \eta_{0}\right)}{\varphi(v)}\left[\frac{w(\sigma(v))}{\Theta(\sigma(v))}\right]^{2} \Delta^{\bar{\alpha}} v \\
& \leq \mathbf{G}\left(\eta, \mathbf{c}_{i}\right) w\left(\mathbf{c}_{i}\right)+\int_{\mathbf{c}_{i}}^{\eta} \frac{\varphi(v)\left[z^{(\bar{\alpha})}(v) \sqrt{\mathbf{G}(\eta, v)}-h_{2}(\eta, v) \Theta(\sigma(v))\right]^{2}}{4 \mathbf{M G}(\eta, v) \Theta(v) e_{\Phi(v)}\left(v, \eta_{0}\right)} \mathbf{G}(\eta, v) \Delta^{\bar{\alpha}} v .
\end{aligned}
$$

Letting $\eta \longrightarrow \overline{\beta_{i}}$, we get

$$
\int_{\mathbf{c}_{i}}^{\bar{\beta}_{i}} \mathbf{G}\left(\bar{\beta}_{i}, v\right)\left\{\frac{b(v) \Theta(v)}{e_{\Phi(v)}\left(\sigma(v), \eta_{0}\right)}-\frac{\varphi(v)\left[z^{(\bar{\alpha})}(v) \sqrt{\mathbf{G}\left(\bar{\beta}_{i}, v\right)}-h_{2}\left(\bar{\beta}_{i}, v\right) \Theta(\sigma(v))\right]^{2}}{4 \mathbf{M G}\left(\bar{\beta}_{i}, v\right) \Theta(v) e_{\Phi(v)}\left(v, \eta_{0}\right)}\right\} \Delta^{\bar{\alpha}} v \leq \mathbf{G}\left(\bar{\beta}_{i}, \mathbf{c}_{i}\right) w\left(\mathbf{c}_{i}\right) .
$$

By multiplying $(\mathbf{G}(v, \eta))$ and taking $\bar{\alpha}$ - fractional integral for (31) from $\eta$ to $\mathbf{c}_{i}$, we get

$$
\begin{aligned}
& \int_{\eta}^{\mathbf{c}_{i}} \mathbf{G}(v, \eta) \frac{b(v) \Theta(v)}{e_{\Phi(v)}\left(\sigma(v), \eta_{0}\right)} \Delta^{\bar{\alpha}} v \\
& \leq-\int_{\eta}^{\mathbf{c}_{i}} \mathbf{G}(v, \eta) w^{(\bar{\alpha})}(v) \Delta^{\bar{\alpha}} v+\int_{\eta}^{\mathbf{c}_{i}} \mathbf{G}(v, \eta) \frac{z^{(\bar{\alpha})}(v) w(\sigma(v))}{\Theta(\sigma(v))} \Delta^{\bar{\alpha}} v \\
& -\int_{\eta}^{\mathbf{c}_{i}} \mathbf{G}(v, \eta) \frac{\mathbf{M} \Theta(v) e_{\Phi(v)}\left(v, \eta_{0}\right)}{\varphi(v)}\left[\frac{w(\sigma(v))}{\Theta(\sigma(v))}\right]^{2} \Delta^{\bar{\alpha}} v \leq-\mathbf{G}\left(\mathbf{c}_{i}, \eta\right) w\left(\mathbf{c}_{i}\right) \\
& +\int_{\eta}^{\mathbf{c}_{i}} \mathbf{G}^{(\bar{\alpha})}(v, \eta) w(\sigma(v)) \Delta^{\bar{\alpha}} v+\int_{\eta}^{\boldsymbol{c}_{i}} \frac{\mathbf{G}(v, \eta) z^{(\bar{\alpha})}(v) w(\sigma(v))}{\Theta(\sigma(v))} \Delta^{\bar{\alpha}} v \\
& -\int_{\eta}^{\mathbf{c}_{i}} \mathbf{G}(v, \eta) \frac{\mathbf{M} \Theta(v) e_{\Phi(v)}\left(v, \eta_{0}\right)}{\varphi(v)}\left[\frac{w(\sigma(v))}{\Theta(\sigma(v))}\right]^{2} \Delta^{\bar{\alpha}} v \leq-\mathbf{G}\left(\mathbf{c}_{i}, \eta\right) w\left(\mathbf{c}_{i}\right) \\
& +\int_{\eta}^{\mathbf{c}_{i}} \mathbf{G}(v, \eta)\left[z^{(\bar{\alpha})}(v)+\frac{h_{1}(v, \eta) \Theta(\sigma(v))}{\sqrt{\mathbf{G}(\eta, v)}}\right] \frac{w(\sigma(v))}{\Theta(\sigma(v))} \Delta^{\bar{\alpha}} v \\
& -\int_{\eta}^{\mathbf{c}_{i}} \mathbf{G}(v, \eta) \frac{\mathbf{M} \Theta(v) e_{\Phi(v)}\left(v, \eta_{0}\right)}{\varphi(v)}\left[\frac{w(\sigma(v))}{\Theta(\sigma(v))}\right]^{2} \Delta^{\bar{\alpha}} v \leq-\mathbf{G}\left(\mathbf{c}_{i}, \eta\right) w\left(\mathbf{c}_{i}\right) \\
& +\int_{\eta}^{\mathbf{c}_{i}} \mathbf{G}(v, \eta) \frac{\varphi(v)\left[z^{(\bar{\alpha})}(v) \sqrt{\mathbf{G}(v, \eta)}+h_{1}(v, \eta) \Theta(\sigma(v))\right]^{2}}{4 \mathbf{M G}(v, \eta) \Theta(v) e_{\Phi(v)}\left(v, \eta_{0}\right)} \Delta^{\bar{\alpha}} v .
\end{aligned}
$$

Letting $\eta \longrightarrow \bar{\alpha}_{i}^{+}$, we get 


$$
\int_{\bar{\alpha}_{i}}^{\mathbf{c}_{i}} \mathbf{G}\left(v, \bar{\alpha}_{i}\right)\left\{\frac{b(v) \Theta(v)}{e_{\Phi(v)}\left(\sigma(v), \eta_{0}\right)}-\frac{\varphi(v)\left[z^{(\bar{\alpha})}(v) \sqrt{\mathbf{G}\left(v, \bar{\alpha}_{i}\right)}-h_{1}\left(v, \bar{\alpha}_{i}\right) \Theta(\sigma(v))\right]^{2}}{4 \mathbf{M G}\left(v, \bar{\alpha}_{i}\right) \Theta(v) e_{\Phi(v)}\left(v, \eta_{0}\right)}\right\} \Delta^{\bar{\alpha}} v \leq-\mathbf{G}\left(\mathbf{c}_{i}, \bar{\alpha}_{i}\right) w\left(\mathbf{c}_{i}\right) .
$$

Divide (22) by $\mathbf{G}\left(\bar{\beta}_{i}, \mathbf{c}_{i}\right)$ and (35) by $\mathbf{G}\left(\mathbf{c}_{i}, \bar{\alpha}_{i}\right)$, we obtain

$$
\begin{aligned}
& \frac{1}{\mathbf{G}\left(\bar{\beta}_{i}, \mathbf{c}_{i}\right)} \int_{\mathbf{c}_{i}}^{\bar{\beta}_{i}} \mathbf{G}\left(\bar{\beta}_{i}, v\right)\left\{\frac{b(v) \Theta(v)}{e_{\Phi(v)}\left(\sigma(v), \eta_{0}\right)}-\frac{\varphi(v)\left[z^{(\bar{\alpha})}(v) \sqrt{\mathbf{G}\left(\bar{\beta}_{i}, v\right)}-h_{2}\left(\bar{\beta}_{i}, v\right) \Theta(\sigma(v))\right]^{2}}{4 \mathbf{M G}\left(\bar{\beta}_{i}, v\right) \Theta(v) e_{\Phi(v)}\left(v, \eta_{0}\right)}\right\} \Delta^{\bar{\alpha}} v \leq w\left(\mathbf{c}_{i}\right), \\
& \frac{1}{\mathbf{G}\left(\mathbf{c}_{i}, \bar{\alpha}_{i}\right)} \int_{\bar{\alpha}_{i}}^{\mathbf{c}_{i}} \mathbf{G}\left(v, \bar{\alpha}_{i}\right)\left\{\frac{b(v) \Theta(v)}{e_{\Phi(v)}\left(\sigma(v), \eta_{0}\right)}-\frac{\varphi(v)\left[z^{(\bar{\alpha})}(v) \sqrt{\mathbf{G}\left(v, \bar{\alpha}_{i}\right)}-h_{1}\left(v, \bar{\alpha}_{i}\right) \Theta(\sigma(v))\right]^{2}}{4 \mathbf{M G}\left(v, \bar{\alpha}_{i}\right) \Theta(v) e_{\Phi(v)}\left(v, \eta_{0}\right)}\right\} \Delta^{\bar{\alpha}} v \leq-w\left(\mathbf{c}_{i}\right) .
\end{aligned}
$$

Adding the above two inequalities, imply a contradiction to hypothesis.

3.3. Oscillation Criteria for Fractional Euler Equation. We consider fractional Euler-type dynamic equation:

$$
\frac{\left(y^{(\bar{\alpha})}(\eta)\right)^{(\bar{\alpha})}+\frac{a}{\eta^{\bar{\alpha}}} y^{(\bar{\alpha})}(\eta)+b}{\eta^{2 \bar{\alpha}}} y(\eta)=h(\eta), \quad \eta \in\left[\eta_{0}, \infty\right)_{\overline{\mathbb{\pi}}}
$$

Theorem 7. Assume that $(-a / \eta) \in \mathscr{R}_{+}$. If there exists $\mathbf{c}_{i} \in\left(\bar{\alpha}_{i}, \bar{\beta}_{i}\right), i=1,2$, a function $\Theta(\eta)>0$ on $[T, \infty)_{\widehat{\pi}}$ and $\mathbf{G} \in \mathscr{G}$, for sufficiently large $T$ such that

$$
\begin{gathered}
\frac{1}{\mathbf{G}\left(\mathbf{c}_{i}, \bar{\alpha}_{i}\right)} \int_{\bar{\alpha}_{i}}^{\mathbf{c}_{i}} \mathbf{G}\left(v, \bar{\alpha}_{i}\right)\left\{\frac{b(v) \Theta(v)}{v^{2 \bar{\alpha}} e_{\Phi(v)}\left(\sigma(v), \eta_{0}\right)}-\frac{\left[z^{(\bar{\alpha})}(v) \sqrt{\mathbf{G}\left(v, \bar{\alpha}_{i}\right)}-h_{1}\left(v, \bar{\alpha}_{i}\right) \Theta(\sigma(v))\right]^{2}}{4 \mathbf{G}\left(v, \bar{\alpha}_{i}\right) \Theta(v) e_{\Phi(v)}\left(v, \eta_{0}\right)}\right\} \Delta^{\bar{\alpha}} v \\
\quad+\frac{1}{\mathbf{G}\left(\bar{\beta}_{i}, \mathbf{c}_{i}\right)} \int_{\mathbf{c}_{i}}^{\bar{\beta}_{i}} \mathbf{G}\left(\bar{\beta}_{i}, v\right)\left\{\frac{b(v) \Theta(v)}{v^{2 \bar{\alpha}} e_{\Phi(v)}\left(\sigma(v), \eta_{0}\right)}-\frac{\varphi(\eta)\left[z^{(\bar{\alpha})}(v) \sqrt{\mathbf{G}\left(\bar{\beta}_{i}, v\right)}-h_{2}\left(\bar{\beta}_{i}, v\right) \Theta(\sigma(v))\right]^{2}}{4 \mathbf{G}\left(\bar{\beta}_{i}, v\right) \Theta(v) e_{\Phi(v)}\left(v, \eta_{0}\right)}\right\} \Delta^{\bar{\alpha}} v>0,
\end{gathered}
$$

then (37) is oscillatory.

Proof. The proof is the same as that of Theorem 6 .

Remark 2. Suppose the conditions in Theorem 5 are replaced by the assumption given as follows:

$$
h(\eta)= \begin{cases}\geq 0, & {\left[\bar{\alpha}_{1}, \bar{\beta}_{1}\right]} \\ \leq 0, & {\left[\bar{\alpha}_{2}, \bar{\beta}_{2}\right]}\end{cases}
$$

then Theorem 5 is accomplished.

Remark 3. In this article, we introduced the interval oscillation criteria for conformable fractional dynamic equations with Riccati transformation. So far, there are many results concerning the fractional model with oscillation criteria (see [19]). However, to the best of our cognizance, there are no results on the oscillation of fractional dynamic equations with forcing term.

\section{Examples}

In this part, in view of the conditions acquired in Section 3, we introduce some examples to represent the adequacy of the suggested nonlinear fractional model and the merits of our methodology.

Example 1. We take the nonlinear conformable fractional damped dynamic equation: 


$$
\begin{aligned}
& \left(\eta^{-1} y^{(1 / 2)}(\eta)\right)^{(1 / 2)}+\eta^{-3} y^{(1 / 2)}(\eta)+\eta^{2} y(\eta)\left(1+e^{y(\eta)}\right) \\
& =\sin \eta, \quad \eta \in[1, \infty)
\end{aligned}
$$

Here, $\widehat{\mathbb{T}}=\mathbb{R} ; \bar{\alpha}=1 / 2 ; \varphi(\eta)=\eta^{-1} ; a(\eta)=\eta^{-3} ; b(\eta)=\eta^{2} ;$ $\mathbf{f}(y(\eta))=(y(\eta))^{\gamma}\left(1+e^{y(\eta)}\right) ; \eta_{0}=1$. Clearly, $\mathbf{f}(y) / y=1+$ $e^{y(\eta)} \geq 1=\mathbf{K}, \sigma(\eta)=\eta, \mu(\eta)=0, \Phi(\eta)=-\eta^{-(5 / 2)}$,

$$
\begin{aligned}
\int_{\eta_{0}}^{\infty} \frac{\Delta^{\bar{\alpha}} v}{\varphi(v)} & =\int_{\eta_{0}}^{\infty} \frac{v^{-(1 / 2)}}{v^{-1}} \mathrm{~d} v=\int_{\eta_{0}}^{\infty} v^{1 / 2} \mathrm{~d} v=\infty, \\
1 & \geq e_{\Phi(\eta)}\left(\eta, \eta_{0}\right)=\exp \left(\int_{\eta_{0}}^{\eta} \Phi(v) \mathrm{d} v\right) \geq 1+\int_{1}^{\eta} \Phi(v) \mathrm{d} v \\
& =1-\int_{1}^{\eta} v^{-(5 / 2)} \mathrm{d} v=1+\frac{2}{3}\left(\eta^{-(3 / 2)}-1\right) \geq \frac{1}{3} .
\end{aligned}
$$

Let $\quad \bar{\alpha}_{1}=n \pi, \quad \bar{\beta}_{1}=\bar{\alpha}_{2}=(n+1) \pi, \quad \bar{\beta}_{2}=(n+2) \pi$, $\mathbf{c}_{i}=\bar{\alpha}_{i}+\bar{\beta}_{i} / 2, i=1,2 ; n=1,2, \ldots, \Theta(\eta)=\eta^{5 / 2}$, and $\mathbf{G}(\eta$, $v)=\eta-v$. Then, we get

$$
\begin{aligned}
& \frac{1}{\mathbf{G}\left(\mathbf{c}_{i}, \bar{\alpha}_{i}\right)} \int_{\bar{\alpha}_{i}}^{\mathbf{c}_{i}} \mathbf{G}\left(v, \bar{\alpha}_{i}\right)\left\{\frac{\mathbf{K} b(v) \Theta(v)}{e_{\Phi(v)}\left(\sigma(v), \eta_{0}\right)}-\frac{\varphi(v)\left[z^{(\bar{\alpha})}(v) \sqrt{\mathbf{G}\left(v, \bar{\alpha}_{i}\right)}+h_{1}\left(v, \bar{\alpha}_{i}\right) \Theta(\sigma(v))\right]^{2}}{4 \mathbf{G}\left(v, \bar{\alpha}_{i}\right) \Theta(v) e_{\Phi(v)}\left(v, \eta_{0}\right)}\right\} \Delta^{\bar{\alpha}} v \\
& \quad+\frac{1}{\mathbf{G}\left(\bar{\beta}_{i}, \mathbf{c}_{i}\right)} \int_{\mathbf{c}_{i}}^{\bar{\beta}_{i}} \mathbf{G}\left(\bar{\beta}_{i}, v\right)\left\{\frac{\mathbf{K} b(v) \Theta(v)}{\boldsymbol{e}_{\Phi(v)}\left(\sigma(v), \eta_{0}\right)}-\frac{\varphi(v)\left[z^{(\bar{\alpha})}(v) \sqrt{\mathbf{G}\left(\bar{\beta}_{i}, v\right)}+h_{2}\left(\bar{\beta}_{i}, v\right) \Theta(\sigma(v))\right]^{2}}{4 \mathbf{G}\left(\bar{\beta}_{i}, v\right) \Theta(v) e_{\Phi(v)}\left(v, \eta_{0}\right)}\right\} \Delta^{\bar{\alpha}} v \\
& \geq \frac{2}{\pi} \int_{\bar{\alpha}_{i}}^{\mathbf{c}_{i}}\left(v-\bar{\alpha}_{i}\right)\left\{v^{9 / 2}-\frac{3 s^{-1}\left[(5 / 2) v^{2} \sqrt{v-\bar{\alpha}_{i}}+\sqrt{v-\bar{\alpha}_{i}} v^{5 / 2}\right]^{2}}{4\left(v-\bar{\alpha}_{i}\right) v^{5 / 2}}\right\} v^{1 / 2} \mathrm{~d} v \\
& \quad+\frac{2}{\pi} \int_{\mathbf{c}_{i}}^{\bar{\beta}_{i}}\left(\bar{\beta}_{i}-v\right)\left\{v^{9 / 2}-\frac{3 s^{-1}\left[(5 / 2) v^{2} \sqrt{\bar{\beta}_{i}-v}+\sqrt{\bar{\beta}_{i}-v} v^{5 / 2}\right]^{2}}{4\left(\bar{\beta}_{i}-v\right) v^{5 / 2}}\right\} v^{1 / 2} \mathrm{~d} v>0 .
\end{aligned}
$$

Hence, by Theorem 5, (40) is oscillatory.

Example 2. We take the nonlinear conformable fractional damped dynamic equation:

$$
\Delta^{(1 / 2)}\left(\eta^{-(1 / 2)} \Delta^{(1 / 2)} y(\eta)\right)+\eta^{-(5 / 2)} \Delta^{(1 / 2)} y(\eta)+\eta^{1 / 2} y(\eta)=\cos 2 t .
$$

Here, $\quad \eta \in[2, \infty)_{\mathbb{Z}} ; \quad \widehat{\mathbb{T}}=\mathbb{Z} ; \quad \bar{\alpha}=1 / 2 ; \quad \varphi(\eta)=\eta^{-(1 / 2) ;}$ $a(\eta)=\eta^{-(5 / 2)} ; b(\eta)=\eta^{1 / 2} ; \eta_{0}=2 ; \mathbf{f}(y(\eta))=y(\eta)$.
Therefore, we get $f^{\prime}(y)=1=\mathbf{M}, \quad \sigma(\eta)=\eta+1$, $\mu(\eta)=1, \Phi(\eta)=-\eta^{-(5 / 2)}, 1+\mu(\eta) \Phi(\eta)=1-\eta^{-(5 / 2)} \geq 1-$ $(1 / 2)>0$ which implies $\Phi(\eta) \in \mathscr{R}^{+}$. Also, 


$$
\begin{aligned}
\int_{\eta_{0}}^{\infty} \frac{\Delta^{\bar{\alpha}} v}{\varphi(v)} & =\int_{2}^{\infty} \frac{v^{\bar{\alpha}-1} \Delta v}{\varphi(v)}=\sum_{v=2}^{\infty} \frac{v^{\bar{\alpha}-1}}{v^{-(1 / 2)}}=\sum_{v=2}^{\infty} 1=\infty \\
1> & e_{\Phi(\eta)}\left(\eta, \eta_{0}\right)=\exp \left(\int_{\eta_{0}}^{\eta} \Phi(v) \Delta v\right) \geq 1 \\
& +\int_{2}^{\eta} \Phi(v) \Delta v=1-\sum_{v=2}^{\eta-1} v^{-(5 / 2)} \\
\geq & 1-\int_{1}^{\eta-1} v^{-(5 / 2)} \mathrm{d} v=1+\frac{2}{3}\left((\eta-1)^{-(3 / 2)}-1\right)>\frac{1}{3}
\end{aligned}
$$

Let $\bar{\alpha}_{1}=n \pi+(\pi / 4), \bar{\beta}_{1}=\bar{\alpha}_{2}=(n+1) \pi+(\pi / 4), \quad \bar{\beta}_{2}=$ $(n+2) \pi+(\pi / 4), \mathbf{c}_{i}=\bar{\alpha}_{i}+\bar{\beta}_{i} / 2, i=1,2 ; n=1,2, \ldots$ and $\Theta$ $(\eta)=\eta^{2}$. Then, all the constraints of Theorem 6 are satisfied with $\mathbf{G}(\eta, v)=\eta-v$, and (40) is oscillatory.

Example 3. We take the following conformable fractional Euler-type dynamic equations

$$
\left(y^{(1 / 2)}(\eta)\right)^{(1 / 2)}+a \eta^{-(1 / 2)} y^{(1 / 2)}(\eta)+b \eta^{-1} y(\eta)=\cos \eta
$$

where $\eta \in[1, \infty)_{\mathbb{Z}}, \widehat{\mathbb{T}}=\mathbb{R}, \bar{\alpha}=1 / 2$, and $\eta_{0}=1$. Then, $\mu(\eta)=$ 0 and $e_{-a / v}\left(\eta, \eta_{0}\right)=\exp \left(\int_{\eta}^{\eta}(-a / v) \mathrm{d} v\right)=\eta^{-a}$ since $\sigma(\eta)=\eta$. If we let $\bar{\alpha}_{1}=n \pi+(\pi / 2), \quad \bar{\beta}_{1}=\bar{\alpha}_{2}=(n+1) \pi+(\pi / 2)$, $\bar{\beta}_{2}=(n+2) \pi+\pi / 2, \quad \mathbf{c}_{i}=\left(\bar{\alpha}_{i}+\bar{\beta}_{i} / 2\right), i=1,2, n=1,2, \ldots$ and $\Theta(\eta)=\eta^{2}$, then all the constraints of Theorem 7 are fulfilled with $\mathbf{G}(\eta, v)=\eta-v$ for suitable choice of $a$ and $b$. Hence, we conclude that equation (45) is oscillatory.

\section{Conclusion}

In this article, we established the interval oscillation criteria for conformable fractional damped dynamic equations with forcing term. The obtained results are improved in the sense which provides sufficient criteria for the forced oscillation of the considered equation with various conditions. Finally, numerical examples are also presented to validate the theoretical results of this study, and in addition, the developed methodology has been tested on the forced oscillation of a fractional Euler-type dynamic equation with an example.

It would be interesting to extend the results proposed in this article to more complex systems, including fractional dynamic equations with time delay. This will be examined in our future works.

\section{Data Availability}

No data were used to support this study.

\section{Conflicts of Interest}

The authors declare that there are no conflicts of interest regarding the publication of this paper.

\section{Acknowledgments}

The fourth author was financially supported by Thailand Science Research and Innovation (TSRI) Grant Fund No. 64A146000001, and the second author was supported by DST-FIST (Grant No. SR/FST/MSI-115/2016).

\section{References}

[1] S. Hilger, "Analysis on measure chains - a unified approach to continuous and discrete calculus," Results in Mathematics, vol. 18, no. 1-2, pp. 18-56, 1990.

[2] M. Bohner and A. Peterson, "̈a," Dynamic Equations on Time Scale, an Introduction with Applications, Birkh, Bostan, MA, USA, 2001.

[3] R. P. Agarwal, M. Bohner, T. Li, and C. Zhang, "Oscillation criteria for second-order dynamic equations on time scales," Applied Mathematics Letters, vol. 31, pp. 34-40, 2014.

[4] Z. Han, T. Li, and S. Sun, "Oscillation for second-order nonlinear delay dynamic equations on time scales," Advances in Differential Equations, vol. 2009, Article ID 756171, 13 pages, 2009

[5] Y. Sui and Z. Han, "Oscillation of second order neutral dynamic equations with deviating arguments on time scales," Advances in Difference Equations, vol. 2018, no. 1, p. 337, 2018.

[6] R. P. Agarwal, M. Bohner, and T. Li, "Oscillatory behavior of second-order half-linear damped dynamic equations," Applied Mathematics and Computation, vol. 254, pp. 408-418, 2015.

[7] M. Bohner and T. Li, "Kamenev-type criteria for nonlinear damped dynamic equations," Science China Mathematics, vol. 58, no. 7, pp. 1445-1452, 2015.

[8] S. H. Saker, R. P. Agarwal, and D. O’Regan, "Oscillation of second order damped dynamic equations on time scales," Journal of Mathematical Analysis and Applications, vol. 330, pp. 1317-1337, 2004.

[9] S. R. Grace, J. Alzabut, S. Punitha, V. Muthulakshmi, and H. Adıgüzel, "On the nonoscillatory behavior of solutions of three classes of fractional difference equations," Opuscula Mathematica, vol. 40, no. 5, pp. 549-568, 2020.

[10] T. Li and S. H. Saker, "A note on oscillation criteria for second-order neutral dynamic equations on isolated time scales," Communications in Nonlinear Science and Numerical Simulation, vol. 19, no. 12, pp. 4185-4188, 2014.

[11] P. ehak, "A critical oscillation constant as a variable of time scales for half-linear dynamic equations," Mathematica Slovaca, vol. 60, pp. 237-256, 2010.

[12] R. Hilfer, Applications of Fractional Calculus in Physics, World Scientific, Singapore, 2000.

[13] I. Podlubny, Fractional Differential Equations, Academic Press, San Diego, CA, 1999.

[14] A. G. M. Selvam, J. Alzabut, R. Janagaraj, and H. Adiguzel, "Oscillation analysis for nonlinear discrete fractional order delay and neutral equations with forcing term," Dynamic Systems and Applications, vol. 29, no. 2, pp. 327-342, 2020.

[15] A. G. M. Selvam, J. Alzabut, M. Jacintha, and A. Ozbekler, "Oscillation results for a class of nonlinear fractional order difference equations with damping term," Journal of Function Spaces, vol. 2020, Article ID 5495873, 10 pages, 2020.

[16] S. G. Samgo, A. A. Kilbas, and O. I. Marichev, Fractional Integral and Derivatives: Theory and Applications, Gordon \& Breach, Yverdon, Switzerland, 1993.

[17] J. Alzabut, S. Manikandan, V. Muthulakshmi, and S. Harikrishnan, "Oscillation criteria for a class of nonlinear 